\title{
Hierarchical Coloured Petrinet Based Healthcare Infrastructure Interdependency Model
}

\author{
N.Nivedita, S.Durbha \\ Centre of studies in resources engineering, IIT Bombay, Powai, Mumbai 400076, India \\ nnivedita@iitb.ac.in, sdurbha@ iitb.ac.in
}

\begin{abstract}
KEY WORDS: Critical Infrastructure Interdependency, Hierarchical Coloured Petrinet, Healthcare Critical Infrastructure, Petri Nets.
\end{abstract}

\begin{abstract}
:
To ensure a resilient Healthcare Critical Infrastructure, understanding the vulnerabilities and analysing the interdependency on other critical infrastructures is important. To model this critical infrastructure and its dependencies, Hierarchal Coloured petri net modelling approach for simulating the vulnerability of Healthcare Critical infrastructure in a disaster situation is studied.. The model enables to analyse and understand various state changes, which occur when there is a disruption or damage to any of the Critical Infrastructure, and its cascading nature. It also enables to explore optimal paths for evacuation during the disaster. The simulation environment can be used to understand and highlight various vulnerabilities of Healthcare Critical Infrastructure during a flood disaster scenario; minimize consequences; and enable timely, efficient response.
\end{abstract}

\section{INTRODUCTION}

Critical Infrastructure (CI) consists of services and technological networks such as healthcare, transport, water supply, electricity supply, information technology etc. These CI's are an essential backbone to ensure effective functioning of society (Panzieri et.al, 2005). There is an interdependency between these critical infrastructures, for example, due to flooding in an area, electricity can get disrupted, underground communication cables get destroyed, this results in the disruption of communication infrastructure (e.g. phone and cell phone services, etc.) and as well as the information technology infrastructure (email services, web services). Disruption in transportation infrastructure (road services, rail services) would further delay the arrival of the repair personnel for getting the underground cables repaired. Hence, to address these issues, a holistic approach is necessary to model the $\mathrm{CI}$ and its dependencies.

A CI can be represented as a set of nodes in a network where they are related to each other through links that shows the relationship among them. These nodes can be interdependent on each other, and interact with each other at various levels, such that the state of each infrastructure influences or is correlated between each other (Pederson et.al, 2006). In this work the Critical Healthcare Infrastructure (CHI) is studied for its ability to respond to disasters such as floods. CHI depends upon a complex interdependent CI network such as transportation, electricity, water supply, Information technology, etc. During disasters it is very vital for $\mathrm{CHI}$ to be protected, accessible and prepared for emergency care. They must be able to provide timely critical services such as medical, nursing care, laboratory, and should have rapid response capabilities. To reduce the consequences of a disaster on the Critical Infrastructure and to ensure a resilient Critical Health Infrastructure network, knowledge, understanding, modelling and analysing the interdependencies between the infrastructures is essential. The aim of this work is to model the interdependencies related to Healthcare Critical Infrastructure using Hierarchical Coloured Petri Nets modelling approach in the event of a flood disaster which is likely to massively disrupt the healthcare infrastructure and the interdependent nodes. Such a model would be useful for understanding the vulnerabilities and develop scenarios for safe and quick evacuation and other rescue operations. Petri net simulation model when integrated with geographic information system can help us to visualize and understand vulnerabilities on a spatio-temporal scale and assess its preparedness and resiliency is healthcare critical infrastructure in the flood disaster scenario.

\section{PETRI NET AND CI INTERDEPENDENCIES}

To model the interdependencies among the Critical infrastructures, recently, Petrinet were used by researchers $t$ model these linkages. Rinaldi et al., 2001 modeled the various elements of urban infrastructure, which include electric power, telecommunications (information and communications), transportation, education, water supply, emergency and government services, and other fundamental systems and services. Gursesli et.al., 2003 applied the graph-based Petri net to identify and examined the interdependencies among the infrastructures due to the failure of power plant as the main supporting infrastructure, the power plant. Krings et.al, 2003 introduced a simple generalized stochastic Petri net model for modelling the cascading failures of critical infrastructures. Immediate and timed events were included in their studies for identifying common-mode faults. Sultana et.al, 2009 modelled flood-related interdependencies among hydroelectricity generating infrastructures using extended Petri net and Markov chain analysis. Omidvar et al., 2014 has performed failure risk assessment of interdependent infrastructure using Petrinet, when an earthquake occurs.

\section{NEED FOR HEALTHCARE INFRASTRUCTURE INTERDEPENDENCY MODEL DURING A DISASTER}

Effective disaster management requires successful operation of complex, interacting human and technological systems. The development of a Coloured Petrinet based simulation model 
would provide new insights, which can be used for policy analysis and training purposes.

Below is an example of a disaster scenario that involves a hospital infrastructure:

"The city has been affected by severe cyclone, the hospital is flooded with water, the hospital emergency department and intensive care units have been impacted the most, rendering the area non-functional. All services in these areas have to be relocated. Main water and power services are been disrupted."

- What is the current flood level? What areas around the hospitals have submerged?

- Is there a failure in one of the Critical Infrastructure (e.g. a substation failure in Electric supply CI), what would be cascading effect on the Healthcare CI?

- How would it prioritize the evacuation process to nearby hospital?

- How would the hospital arrange for emergency evacuation of patients to other hospitals?

\section{METHODOLOGY BASED ON COLORED PETRINET (CPN)}

Petri net is based on graph theory and is used for modelling and analysing the network characteristics, a basic Petri net contains two kinds of nodes: One of the nodes is called as 'place', which describes the states of the system. The other is 'transition' which describes the actions. Places and transitions are connected by directed arcs. The arc expressions describe how the state of the Petri nets change when the transitions occur. Each place contains a set of tokens. The transition fires by removing tokens from its input places and creates tokens, which are distributed to its output places (Petri, 1962) (Murata, 1989) (Bobbio, 1990). However, if the petri net model is quite complex and large, Coloured Petri nets (CPN) can be used to express better than a normal Petri net as CPN can distinguish various places with different colors i.e., by adding a particular data value to a token called as the token colour (Jensen, 1997; Jensen, 1998).

The framework adapted for developing the Hierarchical Coloured Petrinet (HCPN) for Healthcare Critical Infrastructure Interdependency is shown in figure 1, which consists of identifying the interdependent $\mathrm{CI}$, and developing various Coloured Petrinets that are related to the CI network in different subpages of the HCPN.

Integrating the developed model with Geographic Information system

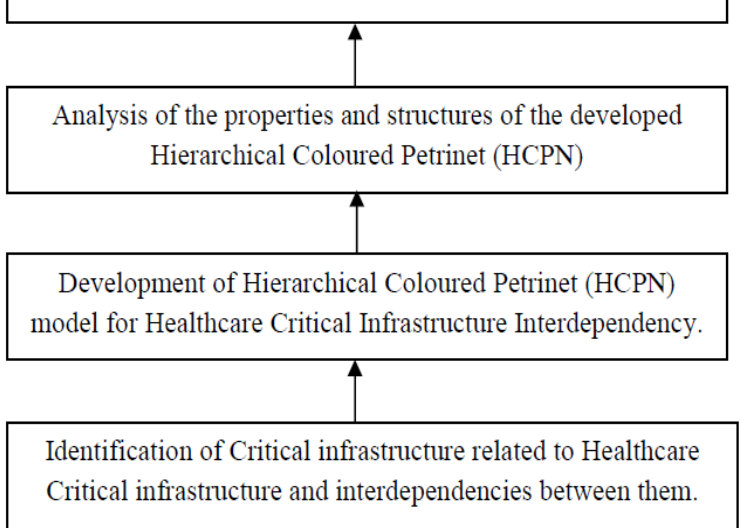

Figure 1.Framework for Healthcare Critical Infrastructure Interdependency model.
The main focus of this work is to construct a HCPN for Healthcare Infrastructure Interdependency, the interaction as well as the influence of Electric supply Infrastructure and Water Supply Infrastructure on Healthcare Infrastructure at the onset of flood. Three different CPN models have been developed, which have been integrated together in a HCPN, such as:

1. Flood simulation CPN.

2. Healthcare Infrastructure Interdependency with Electric Supply and Water Supply Infrastructure CPN.

3. Healthcare Infrastructure CPN.

\subsection{Flood Simulation CPN}

For different flood levels (such as $1 \mathrm{~m}, 2 \mathrm{~m}$, etc.) and different intervals of time $(1 \mathrm{hr}, 2 \mathrm{hr}$.etc.) the Flood simulation CPN subpage snippet as shown in figure 2 would give the Inundation area (IA), Inundation Depth (ID), and flood discharge (FD).

Color set FL represents different flood levels from $1 \mathrm{~m}$ to $20 \mathrm{~m}$ at different intervals of time and is associated by the variable ' $\mathrm{f}$ ', whereas color set Inundation Depth ID gives the Inundation depth for various flood levels considering the inputs from the digital elevation model (DEM) of the area which in turn results in colour set flow discharge (FD); different values such as fd1, $\mathrm{fd} 2, \mathrm{fd} 3, \mathrm{fd} 4$ are the flow discharges, and depending on the flow discharge the geographical area is affected. Flood simulation model provides the input for Healthcare CI Interdependency CPN; the flood affects the Electric supply CI, Water supply CI and Healthcare $\mathrm{CI}$ in that geographical area.

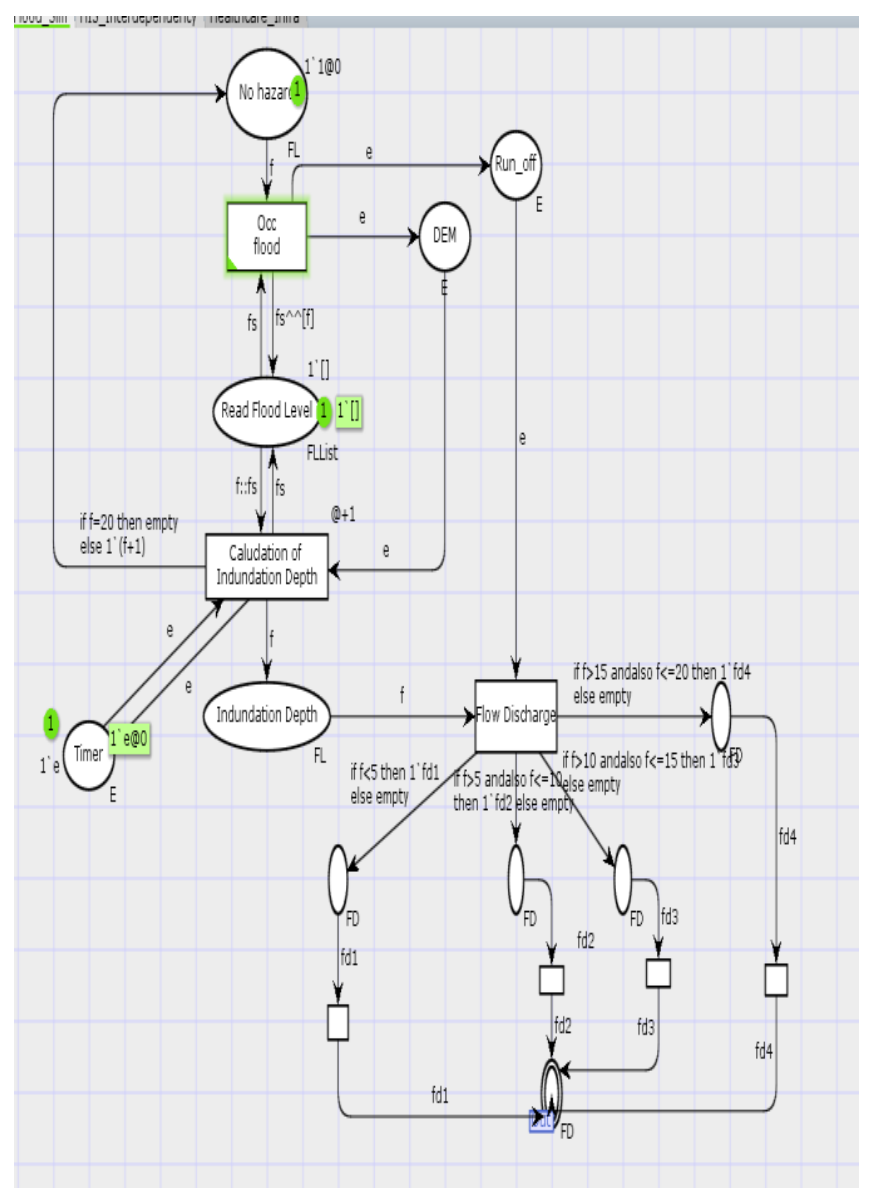

Figure 2. Flood Simulation CPN Model 


\subsection{Healthcare Infrastructure Interdependency CPN}

Healthcare Infrastructure Interdependency $\mathrm{CPN}$ model as shown in figure 3 integrates the following CIs;

1. Water supply CI network model, which consists of pumping stations and storage tanks.

2. Electric Supply CI consisting of a network model of substations located in certain geographical area.

3. Healthcare CI consists of facilities such as Hospitals, ambulances and patients.

From the output obtained from the Flood simulation CPN for different flood level at different time scenarios, the affected nodes (i.e. Hospitals, Electric substations, Water storage tanks, Pumping stations) of the Healthcare CI network; Electric supply CI and Water supply CI respectively can be identified. The various representations of these $\mathrm{CI}$ attributes are as follows:

- Color set ES represents the Electric supply CI, and es1, es2, es3 are different nodes representing the substations and the variable 'es' is associated with the closet ES.

- Color set ST represents the storage tank, and st1, st2, and st 3 represents the different storage tanks nodes.

- Color set PS represents the pumping stations with ps1, ps 2 and ps 3 as the different pumping station nodes.

- Color set HS represents the Healthcare CI such as Hospital, where hs1, hs2, hs3, hs4 represent the various hospital nodes.

Following is a scenario which has been considered while constructing the model:

Disruption of electric substation (es1) would affect the electric substation (es2) which would later affect the working of pumping station (ps1), disruption of which affects the storage tank (st2), which in return affects the water supply for hospital (hs2). Hence, we can see that how there is a cascading effect of failure of one $\mathrm{CI}$ on the other CI.

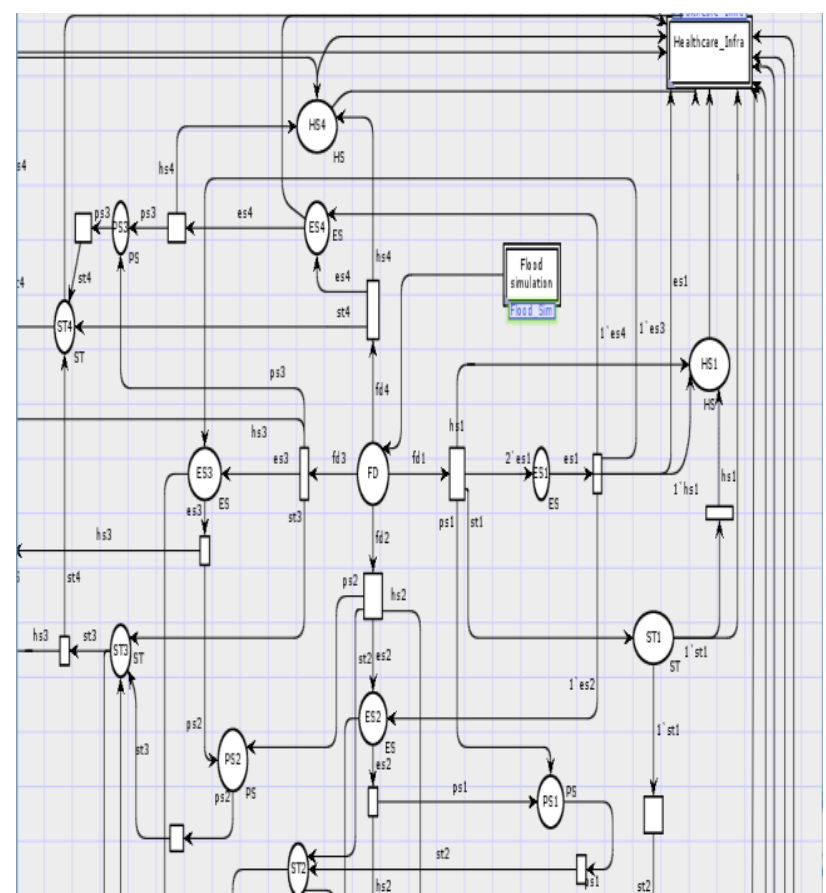

Figure 3. Healthcare CI, Electric Supply CI and Water Supply $\mathrm{CI}$ interdependency HCPN Model.

\subsection{Healthcare Infrastructure CPN}

Healthcare Infrastructure CPN model as shown in the figure 4 is one of the integrated CPN model in Healthcare infrastructure Interdependency HCPN. This CPN model provides us with the activity, which needs to be performed at the affected hospital if there is alarm information indicating the flooding of the hospital (hs1), failure of electric supply (es1), and also failure of water supply (st1) to the hospital. The command centre designated activities would be performed priority wise which includes; Alert and mobilize the Hospital staff, organize the medical equipment for evacuation, alert the ambulance services, alert the patient wards which needs to be evacuated respectively, and later evacuating the patients to nearby unaffected Hospital. For example, if Hospital (hs1) is the affected hospital then the patients can be evacuated to Hospital (hs2). The various color set and the declarations used in Healthcare Infrastructure interdependency HCPN is shown in figure 5.

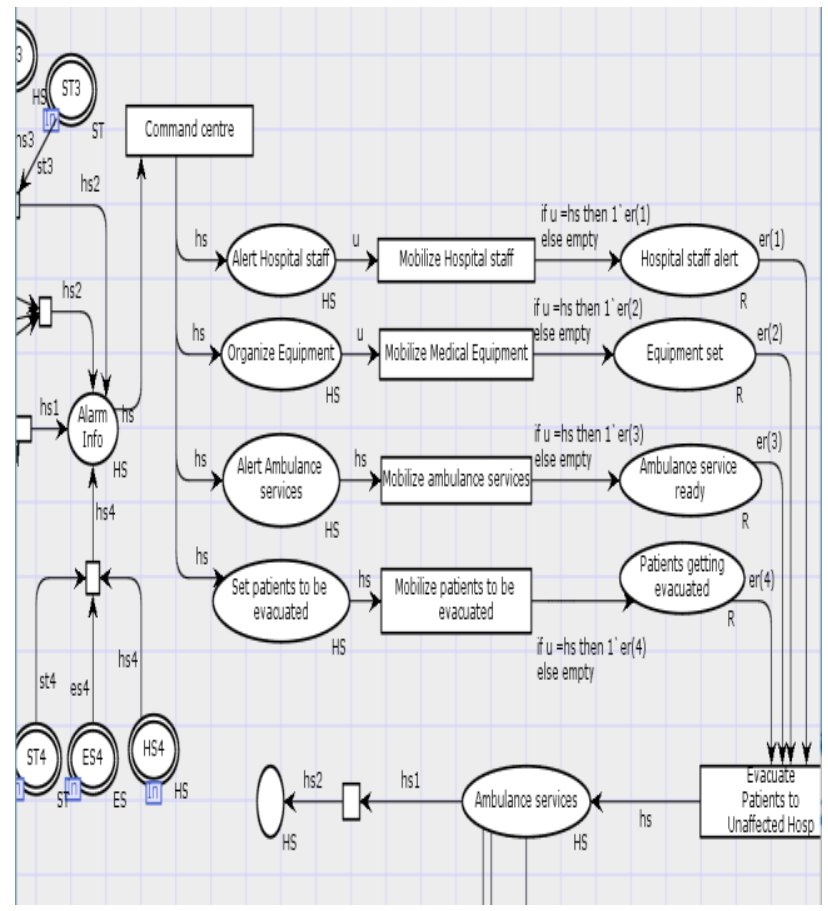

Figure 4. Healthcare CI CPN Model

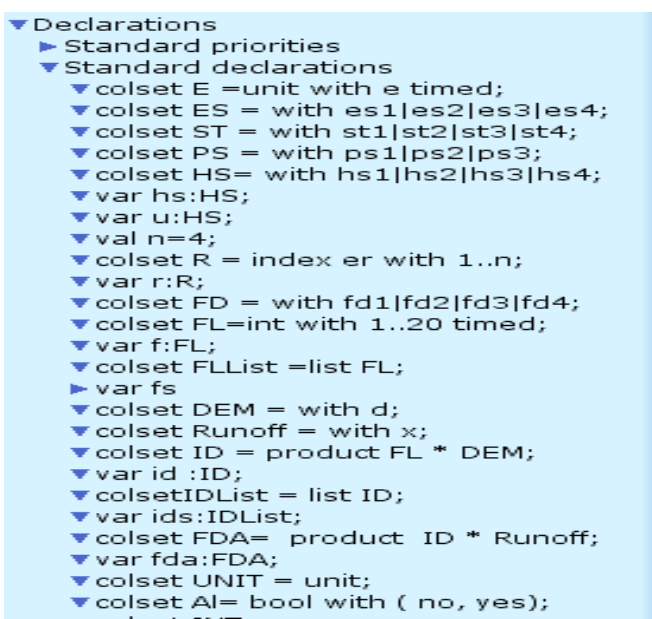

Figure 5. Color set and Declarations Healthcare Infrastructure Interdependency HCPN 
Work is in progress for integrating it with a geographical information system for better visualization and analysing the interdependencies in a geographic analysis environment.

\section{CONCLUSION}

The targeted user community for the proposed model is the disaster response personnel or organisations managing crisis activities (e.g. Health care managers, Emergency management personnel etc.) who are responsible to manage disaster preparedness challenges with a broad range of facility, system, and response activities to quickly inform on and off-duty healthcare, and other interrelated sectors personnel in the event of an emergency.

\section{REFERENCES}

Bobbio A 1990. System modeling with Petri nets. In: Colombia AG, Saiz de Bustamante A (eds) System reliability assessment, pp 102-143.

CPN Tools, http://wiki.daimi.au.dk/cpntools/cpntools.wiki.

Gursesli O, Desrochers AA 2003. Modeling infrastructure interdependencies using Petri Nets.IEEE:1506-1512.

Krings A, Oman P 2003,. "A simple GSPN for modelling common mode failures in critical infrastructures." System Sciences. Proceedings of the 36th Annual Hawaii International Conference on. IEEE, 2003.

Jensen K 1992, "An Introduction to the Practical Use of Coloured Petri Nets", Lectures on Petri Nets II: Applications, Lecture Notes in Computer Science, Vol. 1492, SpringerVerlag, 1998, pp. 237-292.

Jensen K 1997. A brief introduction to coloured petri nets. In Tools and Algorithms for the Construction and Analysis of Systems (pp. 203-208). Springer Berlin Heidelberg.

Murata T 1989. Petri nets: properties, analysis and applications. Proc IEEE 77(4):541-580.

Omidvar, B., Malekshah, M. H., \& Omidvar, H. (2014). Failure risk assessment of interdependent infrastructures against earthquake, a Petri net approach: case study - power and water distribution networks. Natural Hazards, 71(3), 1971-1993.

Panzieri S, Setola R, Ulivi G 2005. "An approach to model complex interdependent infrastructures." World Congress. Vol. 16. No. 1. 2005.

Pederson, P., Dudenhoeffer,D., Hartley.S, and Permann,M 2006. "Critical infrastructure interdependency modeling: a survey of US and international research." (2006): 1-20.

Petri, C. A. 1962. 'Communicating with Automata'. PhD thesis, Technical University Darmstadt,Germany.

Rinaldi SM, Peerenboom JP, Kelly TK 2001. Critical infrastructure interdependencies. IEEE Control System Magazine, 11-25.
Sultana S, Chen Z 2007.Modeling of flood-related interdependencies among critical infrastructures. Geomatics Solutions for Disaster Management. Springer Berlin Heidelberg, 2007. 369-387.

Zuberek W (1991) Timed Petri nets: definitions, properties and applications. Microelectro Reliab 31(4): 627-644. 